\title{
Una Aproximación del Concepto de Empleado Feliz desde la Psicología Positiva
}

\section{An Approximation of the Happy Employee Concept of from Positive Psychology}

Julieta Rebeca Paladines Soto ${ }^{a}$, Silvia Lorena Cuadrado González ${ }^{b}$

\author{
INFORMACIÓN DEL \\ ARTÍCULO \\ Fecha de recepción: 20 de febrero del \\ 2017 \\ Fecha de aceptación: 15 de junio de \\ 2017
}

\footnotetext{
${ }^{a}$ Psicóloga Organizacional, Universi dad Católica Santiago de Guayaquil Ecuador.

E-mail: jrpaladines@uees.edu.ec
}

\footnotetext{
Psicóloga Clínica, Universidad Católica Santiago de Guayaquil Ecuador. Docente de la Universidad Espíritu Santo - Ecuador.

E-mail silviacg@uees.edu.ec
}

\begin{abstract}
Resumen
Las compañías actualmente se enfrentan a un mercado muy competitivo y necesitan contar con empleados comprometidos a desarrollar estrategias desafiantes. De acuerdo a las investigaciones, los empleados felices son más eficientes y comprometidos. Con ello surge la pregunta ¿Cuál es el concepto de empleado feliz? En la revisión bibliográfica realizada no se encuentra un concepto de "empleado feliz" por lo cual éste artículo tiene como objetivo analizar los conceptos de bienestar, felicidad y psicología positiva para encontrar una aproximación a éste concepto. Se realiza una revisión con el método hermenéutico, los orígenes del concepto de bienestar y felicidad hasta los estudios actuales de psicología positiva. Se concluye con tres aproximaciones al concepto de empleado feliz.
\end{abstract}

\section{Palabras Clave:}

Empleado feliz, bienestar, felicidad, psicología positiva, flujo.

\section{Clasificación JEL: M1}

\begin{abstract}
Companies currently face a very competitive market and need to have employees who are committed to developing challenging strategies. According to research, happy employees are more efficient and committed. Because of this, we ask ourselves what the concept of a happy employee is. The "happy employee" concept was not found in the bibliographic review so that, in order to find an approximation to the concept of happiness, this article aims to analyze the concepts of well-being, and positive psychology. A methodological revision looks into the origin of the concepts of well-being and happiness all the way to positive psychology studies. This paper concludes with three approaches to the concept of a "happy employee".
\end{abstract}

\section{Keywords}

Happy employee, well-being, happiness, positive psychology, flow.

JEL Classification: M1

42

PODIUM No. 31, Guayaquil, Junio 2017, pp. 42-53

Universidad Espíritu Santo - UEES

ISSN: $1390-5473$ 


\section{Introducción}

En la actualidad las empresas presentan un alto interés en la felicidad de los empleados dada la creciente demanda de organizaciones eficientes y competitivas, por ello contar con el mejor recurso humano resulta más que nunca importante (Salanova \& Schaufeli, 2009). La felicidad es un concepto que se ha discutido desde hace muchos siglos atrás con Aristóteles como el filósofo más destacado, pero no es sino hasta hace pocas décadas cuando se le ha prestado especial atención con el surgimiento de la psicología positiva.

De hecho, existe a nivel mundial desde el 2012, el índice de felicidad de los países mediante un estudio solicitado por la Organización de Naciones Unidas (ONU) donde el puntaje es dado con base en datos del Producto Interno Bruto (PIB) per cápita, percepción de libertad de sus habitantes, esperanza de vida o el nivel de servicios de la nación (Blastingnews, 2015). A nivel latinoamericano existen ya en varios paises estudios relevantes en materia de bienestar, felicidad, felicidad en el trabajo y flujo. Los países de México (Salazar \& Arenas, 2016), Venezuela (Millán \& Aubeterre, 2013) y Ecuador (López \& Fierro, 2015) presentan importantes avances.

Ahora bien, la revisión de literatura permite identificar la falta de un concepto del empleado feliz, por lo que este artículo tiene como objetivo principal realizar una primera aproximación del concepto de empleado feliz. A través de una revisión teórica de los conceptos de bienestar, felicidad, psicología positiva, flujo y engagement se establece una aproximación al concepto e identifica la relación entre estas variables y el empleado feliz. El método a utilizar es el hermenéutico dado que se realizarán revisiones conceptuales de los principales autores para intentar tener una aproximación a un nuevo concepto y no se realizarán comprobaciones empíricas.

\section{Revisión de la Literatura}

Actualmente las empresas se enfrentan a grandes presiones mundiales por un crecimiento rápido con el menor recurso posible. Así que los empleados con los que cuentan deben ser los idóneos y estar comprometidos física y psicológicamente con la compañía para alcanzar las metas propuestas. Es por ello, que el empleado es más importante ahora que en el pasado (Salanova y Schaufeli, 2009). Pero ¿qué hace que un empleado esté comprometido con una empresa? ¿Qué determina la felicidad en él? Para elaborar el constructo del empleado feliz se deben revisar algunos conceptos importantes como: bienestar - felicidad, psicología positiva - engagement y flujo.

\section{Bienestar y Felicidad.}

El concepto de bienestar ha sido ampliamente revisado desde siglos antes de Cristo. Varios filósofos como Platón realizaron algunos escritos pero no fue sino Aristóteles en su Ética Nicomaquea (1098 b) quien describió el concepto de una manera más sistemática. Para él los bienes se clasifican en: externos (materiales), psíquico o del alma (sentirse bien) y 
físicos (salud), les coloca una jerarquía y determina que en primer lugar está el bienestar del alma, en segundo y tercer lugar los bienes externos y físicos pero éstos cobran sentido únicamente como medios para alcanzar el bienestar del alma (Yakubova, 2016). Entonces, para estar bien es necesario, según Aristóteles, poseer estos tres bienes (Valdés, 1991).

De la misma manera la Organización mundial de la Salud (OMS) el 7 de abril de 1948 declara que la salud es un estado de completo bienestar físico, social y mental y no se limita únicamente a la ausencia de afecciones o enfermedades (OMS, 2016). Asimismo Sen (1998) sostiene que para evaluar el bienestar de una persona se debe poner especial atención al capital humano, conjunto de habilidades que tiene una persona para ser productivo, y la capacidad humana, libertad de la persona a elegir cómo usar sus habilidades en bien de una sociedad.

Ahora bien, la definición de felicidad también tiene sus orígenes en la época de Aristóteles, quien aseguraba que la finalidad de cualquier persona es siempre la búsqueda de la felicidad y que era el bien supremo del hombre pero, a diferencia del bienestar físico y material, lo que se conoce hoy de felicidad no ha variado mucho desde Aristóteles y es apenas en los últimos años donde empieza a tener un lugar en las investigaciones científicas (Csikszentmilhayi, 1997). Si se revisan las publicaciones sobre felicidad de 1990 eran cero al año y en el 2011 llegaron a ser casi treinta mil (Beytía y Calvo, 2011).
Uno de los autores que ha dedicado muchos años a su investigación es Csikszentmihalyi, para él hay dos formas principales para concebir la felicidad: El primero de ellos es como un rasgo personal, o una disposición relativamente permanente para experimentar el bienestar, independientemente de las condiciones externas y la segunda es considerar como un estado, o una experiencia subjetiva transitoria que responde a eventos o condiciones momentáneas del medio ambiente (Csikszentmihalyi \& Mei-Ha, 1991).

Pero qué hace feliz a las personas? Varios estudios realizados por Csikszentmihalyi en los años 80 hablaban de algunos factores como la libertad y los psicólogos han asumido que, en general, la experiencia de la libertad es una atribución que las personas hacen acerca de su comportamiento en determinadas condiciones (Csikszentmihalyi, 1980). Eso puede explicar que las actividades de ocio o el juego son un claro ejemplo de momentos de felicidad (Csikszentmihalyi, 1975). Pero también depende en muchas ocasiones de aprender a disfrutar de las cosas que se encuentran en el día a día de las acciones, no pensar en la felicidad como algo que se tendrá algún día cuando por ejemplo: tenga un mejor empleo, tenga un hijo o tenga más dinero (Fuentes, 2015).

En el año 2005 Sonja Lyubomirsky junto a Ken Sheldon y David Schkade realizaron una investigación científica para identificar los determinantes de la felicidad (Lyubomirsky, 2005) cuyos resultados se explican en la figura 1:

44

PODIUM No. 31, Guayaquil, Junio 2017, pp. 42-53

Universidad Espíritu Santo - UEES

ISSN: $1390-5473$ 


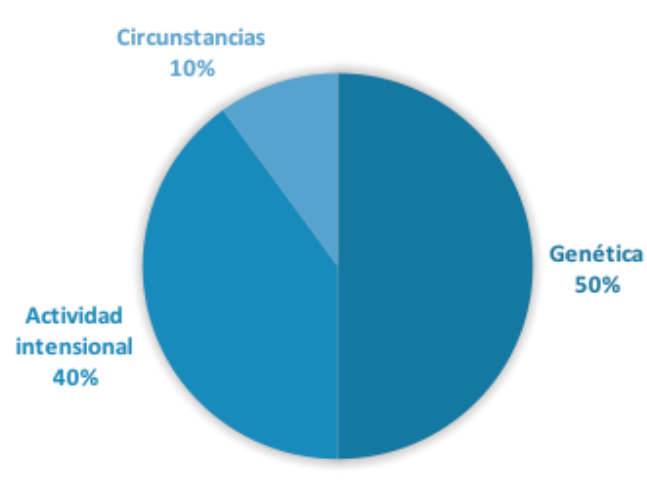

Figura 1. Determinantes de la felicidad. (Lyubomirsky, 2005)

Como se aprecia en la figura 1, el 50\% de la felicidad está determinada por la carga genética de la persona, el punto referencial, y ésto se ha podido demostrar en casos de gemelos idénticos separados al nacer, es como el peso o los índices de colesterol que, cuando existe una predeterminación genética, siempre hay niveles fijos que no pueden ser reducidos. Otro de los resultados que reflejó este estudio fue que aunque parezca increible solo el $10 \%$ de la felicidad está determinada por las circunstancias o situaciones objetivas como el dinero, la salud, la belleza física, estado civil, etc.

Esto quiere decir que si a un grupo de personas se les diera la misma casa y la misma cantidad de dinero la diferencia de felicidad sería apenas del 10\%. ¿y el $40 \%$ restante? Este porcentaje es el determinado por el comportamiento intencional. Por lo tanto, la felicidad no se busca tratando de cambiar los genes o buscando cambiar las circunstancias como el dinero o los bienes materiales, sino potencializando ese $40 \%$ que está al alcance de todo ser humano. De hecho, si bien la depresión tiene una carga genética, de- pende de cada individuo que ésta se ponga en manifiesto e influya positiva o negativamente en nuestras vidas (Lyubomirsky, 2005).

Entonces, ¿Cómo potencializar la felicidad? La respuesta está, según las investigaciones, en conocer las características de las personas felices y sus prácticas para poder adoptarlas en nuestra vida (Salanova \& Schaufeli, 2009):

- Sesgo Positivo: Ver las cosas de una manera más positiva de lo que realmente son y buscar mejorar su felicidad constantemente;

- Invulnerabilidad a la comparación social: No comparar su situación con otros para identificarse más o menos felices;

- Toma de decisiones adaptativas: Tomar decisiones más rápidas y adaptativa, si se enfrenta a múltiples opciones escoger rápidamente sin preguntarse continuamente si las otras pudieron ser mejores y

- Falta de insistencia intrusiva: No detenerse excesivamente a evaluar sus errores, no lamentarse por mucho tiempo.

Es importante en este artículo dar un espacio al concepto de Psicología positiva dado que es la ciencia que se ha preocupado por el estudio de la felicidad en los últimos veinte años.

\section{Psicología Positiva.}

La psicología tradicional ha enfocado sus estudios en las patologías o neurosis del ser humano por muchos años, con base en ellos ha ayudado a desarrollar varios de los descubrimientos que hoy se 
conocen y los tratamientos y terapias; sin embargo, poco se había puesto atención en las características de las personas felices y cómo mediante el desarrollo de e llas ayudar a mantener el bienestar de las $p$ ersonas (Zúñiga, 2008).

Así nace la psicología positiva, que no es otra cosa que una rama de la psicología, que, con la misma rigurosidad científica que está focaliza su atención en un campo de investigación e interés distinto al adoptado tradicionalmente: las cualidades y características positivas humanas (Vera, 2006).

Como se ha revisado en este artículo, el concepto de felicidad tiene muchos siglos de historia pero fue Martin Seligman en el año 2000, en su discurso como presidente de la American Psychological Association, quien cuestionó por primera vez el enfoque negativo que las investigaciones psicológicas habían tenido hasta el momento, más adelante aseguraría que el objetivo de este nuevo movimiento se basaría en tres pilares: el estudio de las emociones positivas; el estudio de los rasgos positivos (foco en fortalezas, virtudes y habilidades) y el estudio de las instituciones positivas (democracia, familias, entre otras) (Seligman, 2005).

Es importante mencionar que con este nuevo enfoque, Seligman ha recibido tanto elogios como críticas que aseguran que la Psicología positiva no es una ciencia, que no aporta conceptos nuevos, que carece de soporte científico y que la mayoría de conceptos se han analizado anteriormente bajo otros nombres (Prieto-Ursúa, 2006). Se puede encontrar de hecho algunos artículos del mismo Mihali Csikszentmihalyi en 1969 en el estudio de la conducta de las personas en la vida cotidiana y la experimentación de emociones positivas (Arias, 2014) sin embargo, es la psicología positiva la que abarca todos estos temas bajo los pilares mencionados por Seligman.

En línea con lo anterior la Psicología debe ser capaz de ayudar a documentar qué tipo de familias resultan en niños felices, qué trabajo obtienen la mayor satisfacción entre los trabajadores y cómo la vida de las personas pueden ser más digna de ser vivida (Seligman, 2005). El objetivo de la psicología positiva es comenzar a generar un cambio que lleve del enfoque de la psicología de la preocupación al enfoque del bienestar, la alegría y la satisfacción (del pasado); la esperanza y el optimismo (del futuro); y el flujo y la felicidad (del presente)(Csikszentmihalyi y Seligman, 2000).

Existen además algunos contextos del ser humano donde resulta importante evaluar la capacidad de ser feliz como la familia, las instituciones educativas y el trabajo.

\section{Felicidad en el trabajo.}

Es importante, luego de revisar los conceptos de felicidad, bienestar y psicología positiva, hacer un análisis de los factores que pueden ayudar a un empleado a sentirse feliz en su lugar de trabajo bajo la perspectiva de la psicología positiva, para ello se analizarán dos conceptos ampliamente desarrollados en los últimos años como gestores de la felicidad en el 
trabajo: el engagement y la teoría del flow.

Hablar de felicidad no parece estar relacionado al trabajo, pues el trabajo es algo serio y la felicidad algo no tan serio (Marks, 2015). De hecho según investigaciones realizadas en los años ochenta, existe una relación entre los índices de felicidad y los días de la semana siendo el sábado el día con índices más altos de felicidad y los domingos más bajos, esto por la proximidad a la jornada laboral. (Csikszentmihalyi, 1980). Pero porqué los trabajadores verían el regreso al trabajo como algo no tan feliz?

En este artículo se ha revisado que solo el $40 \%$ de la felicidad está determinado por acciones intencionales de las personas y que el $10 \%$ puede estar influenciado por situaciones externas, bajo esta premisa las empresas pueden influenciar solo en un 10\% el sentimiento de felicidad de un empleado. Sin embargo, algunas investigaciones dan como resultado que existen empresas con mayor número de empleados felices que otras, cuya rotación es menor y con índices de engagement más altos y hay estudios que demuestran que las personas que se sienten felices en su trabajo llegan más temprano, se enferman menos y son más productivos. Esto cambia la ecuación de éxito igual felicidad, pues se conoce que aquellos empleados que se enfocan en su felicidad son doblemente exitosos que aquellos que no, entonces la felicidad lleva al éxito y no al contrario (Marks, 2015) pues la felicidad es un estado psicológico necesario que todo trabajador debe alcanzar y desarrollar si su finalidad es el éxito (Cabanas \& Sánchez-González, 2016).

Como se citó previamente acerca de lo que pasa en el interior de las personas felices, en los empleados felices se ha encontrado que éstos:

1. Interpretan su trabajo, su desempeño, sus compañeros y ambiente laboral de una manera positiva.

2. No se comparan en términos de desempeño con sus compañeros.

3. Ante problemas laborales no se detienen en los errores y desvían su atención de forma positiva.

El término que mejor encierra éstas y otras características positivas en el trabajo es el engagement, considerado como un estado cognitivo-afectivo experimentado por el empleado persistente en el tiempo con tres características: vigor, dedicación y absorción. Ésta última al igual que el flujo se define como un estado de concentración y disfrute sin determinar el paso del tiempo pero se caracteriza por su persistencia (Salanova, Schaufeli, Llorens, Peiro, \&Grau, 2000).

Las características del engagement son tres: vigor, energía positiva que experimenta el empleado en el ejercicio de su labor; dedicación, nivel de entusiasmo que expresa el empleado por la importancia que siente en el ejercicio de su trabajo y absorción, nivel alto de concentración, percepción de que el tiempo pasa sin que el empleado lo note por su nivel de concentración en la tarea (Fernández \& Merino, 2016). Estas características hacen que el empleado comprometido se sienta feliz en su lugar de trabajo, sea 
más leal a la organización y piense menos en abandonarla (Orgambídez-Ramosa, Pérez-Morenoc, \& Borrego-Alés, 2015).

Ahora, las actividades que influyen de manera positiva al incremento del engagement en los empleados según varios años de investigaciones (Ocampo Bustos, Juárez García, Arias Galicia, \& Hindrichs, 2015) son: el apoyo social de jefes y compañeros de trabajo, la retroalimentación positiva del desempeño, la autonomía elevada en el cargo, la variedad de tareas, la capacitación y las oportunidades de desarrollo.

En los estudios mencionados anteriormente sobre el $40 \%$ del nivel de felicidad influenciable de manera intencional se encuentra que la práctica de actividades características de las personas felices puede potencializar positivamente los índices de felicidad, si esto es llevado a la parte laboral se puede explicar mediante el siguiente cuadro su aplicación positiva:

Tabla 1.

Prácticas de personas felices aplicadas al trabajo

\begin{tabular}{|c|c|}
\hline $\begin{array}{l}\text { Prácticas de las personas } \\
\text { felices. }\end{array}$ & Aplicación al trabajo \\
\hline Cuidar cuerpo y alma & $\begin{array}{l}\text { Políticas claras de salud y campañas } \\
\text { sobre la necesidad de descanso } \\
\text { adecuado. }\end{array}$ \\
\hline $\begin{array}{l}\text { Identificación de las } \\
\text { fortalezas }\end{array}$ & $\begin{array}{l}\text { Robusto plan de desarrollo que } \\
\text { identifique talento y brinde feedback. }\end{array}$ \\
\hline Sesgo positivo & $\begin{array}{l}\text { Crear ambientes positivos de trabajo y } \\
\text { lenguaje evitando términos negativos. }\end{array}$ \\
\hline $\begin{array}{l}\text { Fortalecer las relaciones } \\
\text { sociales }\end{array}$ & $\begin{array}{l}\text { Incentivar prácticas sociales entre } \\
\text { empleados, trabajo en equipo y } \\
\text { colaboración responsable. }\end{array}$ \\
\hline
\end{tabular}

Como se observa en la Tabla 1, las prácticas de las personas felices pueden ser llevadas a la práctica al lugar de traba- jo si la empresa está interesada en mejorar los niveles de engagement de sus empleados y así aumentar su felicidad.

En línea con lo anterior es importante mencionar que lo contrario a engagement se llama burnout y es el síndrome que lleva aun empleado a sentirse quemado en su trabajo. Para prevenir el burnout y mantener el engagement se deben analizar las demandas y recursos con los que cuenta un empleado. Las demandas de un cargo pueden ser físicas o psíquicas y son positivas en la medida en la que la persona cuente con los recursos necesarios para responder a dichas demandas. Los recursos son entendidos como los aspectos físicos, sociales u organizacionales que permiten al empleado responder adecuadamente a las demandas solicitadas. Cuando los recursos son escasos las demandas pueden convertirse en agentes estresores y llevar al empleado a niveles de estrés y luego burnout (Montoya \& Moreno, 2012), por el contrario cuando las demandas y los recursos están equilibrados el empleado puede experimentar niveles de engagement (Moreno, Corso de Zúñiga, Sanz-Vergel, Rodriguez \& Boada, 2010).

Otros estudios aseguran que la felicidad se incrementa en la medida en que los individuos cuentan con los medios para aprender habilidades que se pueden implementar para enfrentar los desafíos razonables; acompañados de libertad para expresarse dentro de los límites de la responsabilidad; que les permite experimentar la alegría de la interacción con sus compañeros (Csikszentmihalyi \& Hunter, 2003).

48

PODIUM No. 31, Guayaquil, Junio 2017, pp. 42-53

Universidad Espíritu Santo - UEES

ISSN: 1390 - 5473 
Otro término importante en esta revisión es el Flow: la teoría del flujo fue creada por Csikszentmihalyi cuando revisaba un análisis del juego y encontró que hay un estado experiencial común que está presente en diversas formas de juego, y también bajo ciertas condiciones en otras actividades. El Flujo denota la sensación holística presente cuando se actua con total implicación. Se experimenta como una sensación unificada que fluye de un momento a otro, en el que se siente en control de las acciones, y en el que hay poca diferencia entre el yo y el medio ambiente; entre el estímulo y la respuesta; o entre el pasado, presente y futuro (Csikszentmilhayi, 1997).

Llevado a la parte laboral el flujo se experimenta en gran cantidad cuando el trabajador se ve expuesto a un reto fuerte que demanda de él un desafío en sus habilidades pero es consciente de que lo puede hacer, aquí puede ser que tenga que renunciar a una cierta cantidad de placer para realizar tareas complejas, pero no renuncia al goce del logro alcanzado (Csikszentmihalyi, 2002). Para experimentar esta sensación máxima de disfrute, la persona debe haber usado al máximo sus habilidades y el desafío debe llegar también a su punto máximo. En el gráfico 2 se puede explicar las diferentes fases de interacción entre desafío y habilidades.

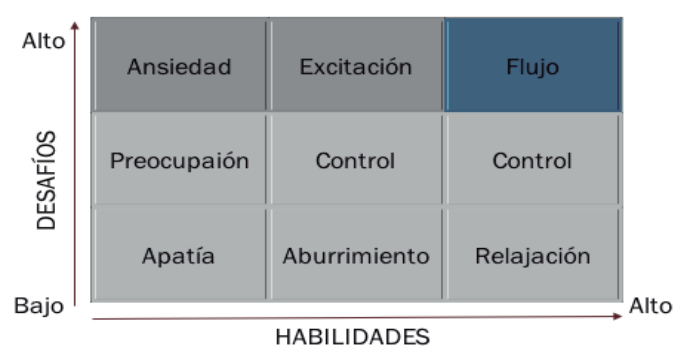

Figura 2. Experiencia del flujo. (Csikszentmilhayi, 1997, p. 44).

En varios estudios realizados en relación al flujo en diferentes profesiones los encuestados informaron de una experiencia subjetiva muy alta que disfrutaron tanto que estaban dispuestos a ir más lejos para experimentarla de nuevo. Esa es la 'experiencia de flujo', también utilizaron la metáfora de una corriente que los empujó a lo largo de esfuerzo (Csikszentmilhayi, 1997).

Para finalizar es importante mencionar que todos las empresas donde el engagement y el flujo han sido experimentados y arrojado resultados positivos han contado con líderes que apuestan por implementar éstas prácticas (Goleman, 2005). Estos líderes apuestan por cultivar el capital psicológico de sus empleados, entendido como el conjunto de factores y procesos que permiten aprender a sobrevivir y a desarrollar fortalezas personales para sobrellevar adversidades (Casullo, 2009) cuyas características son: la autoeficacia, el optimismo, la esperanza y la resiliencia (Contreras, 2011). Además existe una relación directa entre capital psicológico y el desempeño, el ausentismo, la empleabilidad, la satisfacción y compromiso (Delgado y Castañeda, 2011) 
así como el nivel de servicio (Martínez, Moliner, Ramos, Luque, y Gracia, 2014).

\section{Conclusiones}

Luego de la revisión bibliográfica del presente paper las conclusiones son:

De acuerdo con el objetivo principal de este artículo en el concepto de felicidad se puede concluir en primer lugar que éste concepto forma parte del concepto de bienestar y a pesar de que se lo considera un bienestar subjetivo, en las investigaciones sobre los determinantes de la felicidad existen factores externos que influyen sobre la misma como son la salud (bienestar físico) y el dinero (bienestar material, entonces como aseguraba Aristóteles los tres bienes se complementan siendo la felicidad el bien supremo.

En cuanto al método hermenéutico, luego de las revisiones conceptuales de los principales autores de bienestar, felicidad, psicología positiva y engagement, se concluye que es un método válido para la construcción de un nuevo concepto ya que permitió revisar las diferentes corrientes y encontrar el origen de conceptos como bienestar desde la filosofía Aristotélica, saber que es el origen del concepto de felicidad y mediante la revisión de conceptos de felicidad encontrar su conexión con la psicología positiva y finalmente su aplicación en el trabajo y medición de engagement.

En cuanto a la utilidad de esta revisión para la gestión de Talento Humano, el aporte más importante está en los determinantes de la felicidad, pues se otorga un $10 \%$ a factores externos donde entraría el lugar de trabajo, sin embargo trabajar en ese $10 \%$ daría una ley parecida a la ley de pareto donde las empresas pueden potencializar ese $10 \%$ e influenciar positivamente en el $40 \%$ de felicidad intencional que corresponde al empleado, potencializando sobretodo actividades que lleven al empleado a practicar actividades que caracterizan a la gente feliz.

En el auge del estudio de la felicidad encontré limitaciones en el análisis de bienestar integral dado que las empresas miden los niveles de felicidad mediante el engagement y no los factores de bienestar físico y materiales, esto es: cómo se aseguran las compañías en la parte de salud no solo por la ausencia de síntomas sino en el mejoramiento de la misma y en el caso de los bienes materiales cómo se aseguran que éstos bienes son los básicos que cada empleado necesita para estar "bien".

Una primera aproximación al concepto de empleado feliz, tomando en cuenta el bienestar como concepto global que abarca la felicidad, es el siguiente: Empleado feliz: Aquel empleado que experimenta en el desempeño de su labor libre y voluntaria una sensación de bienestar integral que puede potencializar con el pasar del tiempo.

Otra aproximación desde los estudios de los determinantes de la felicidad es: Empleado feliz: Aquel empleado que logra en el desempeño de su labor libre y voluntaria incrementar su punto basal de

50

PODIUM No. 31, Guayaquil, Junio 2017, pp. 42-53

Universidad Espíritu Santo - UEES

ISSN: $1390-5473$ 
de felicidad.

Una última aproximación desde el análisis de la psicología positiva es: Empleado feliz es aquel empleado que en el desempeño de su labor libre y voluntaria experimenta sensaciones de flujo y engagement constantes.

Finalmente es importante mencionar que los estudios hechos para experiencias de flujo o engagement se han realizado en países americanos y Europeos pero no se encuentran este tipo de análisis en países asiáticos, por ejemplo en plantas de China) donde sería importante saber la relación de engagement y flujo entre sus empleados. Es necesario realizar investigaciones futuras de los factores que influyen en la felicidad de un empleado en diferentes países, continentes, cultura, reformas laborales, entre otras variables para identificar si se da la relación de $50 \%$ genética, $40 \%$ intencional y $10 \%$ influenciable y si éste último puede variar según éstos factores.

\section{Referencias}

Arias, W. (2014). Conducta prosocial y Psicología positiva. UNIFE, 38-39.

Blastingnews(2015). Los 10 países más felices del mundo (Naciones Unidas). Obtenido de http://es.blastingnews.com: http://es.blastingnews.com/ocio-cultura/2015/04/1os-10-pai ses-mas-felices-del-mundo-nacion es-unidas-00371807.html
Cabanas, E., \& Sánchez-González, J.-C. (2016). Inverting the pyramid of needs: Positive psychology's new order for labor success. Psicothe$m a, 28$, No. 2, 107-113 .

Casullo, M. (2009). Capital psiquico: aportes de la psicología positiva. Psicodebate 6. Psicología, cultura y sociedad, 59-72.

Company, C. C. (2008). www.cocacola.es. Obtenido de www.cocacola.es.

Contreras, F. (2011). La gestión del capital psicológico. Artículo de revista web económica. Bogotá, Colombia: Revista Dinero.

Csikszentmihalyi, M. (1975). Play and Intrinsic Rewards. Flow and the Foundations of Positive Psychology, 135-142.

Csikszentmihalyi, M. (1980). The Experience of Freedom in Daily Life. Flow and the Foundations of Positive Psychology The Collected Works of Mihaly Csikszentmihalyi, 55-67.

Csikszentmihalyi, M., \& Mei-Ha, M. (1991). The Situational and Personal Correlates of Happiness: ACross-NationalComparison. Flow and the Foundations of Positive Psychology, 69-81.

Csikszentmilhayi, M. (1997). Fluir, una psicología de la felicidad. Madrid.

Csikszentmihalyi, M., \& Seligman, M. (2000). Positive Psychology: An Introduction. 
Flow and the Foundations of Positive Psychology, 279-297.

Csikszentmihalyi, M. (2002). The Concept of Flow. Springer, 239-269.

Csikszentmihalyi, M., \& Hunter, J. (2003). Happiness in Everyday Life: The Uses of Experience Sampling. 89-101.

Csikszentmihalyi, M. (2014). Toward a Psychology of Optimal Experience . Flow and the Foundations of Positive Psychology, 209-227.

Delgado, L., \& Castañeda, D. (2011 ). Relación entre capital psicológico y la conducta de compartir conocimiento en el contexto del aprendizaje organizacional . Acta colombiana de Psicología, 61-70.

Fernández-Arata, M., \& Merino-Soto, C. (2016). El Lado Socialmente Deseable de las Respuestas a Medidas de Burnout y Engagement: un Estudio Preliminar. Revista Colombiana de Psicología, 25(1), 83-94.

Fuentes, N. (10 de 03 de 2015). La ciencia de la felicidad aplicada en el trabajo. Foro Internacional. Quito, Ecuador.

Goleman, D. (2005). Liderzgo que obtiene resultados. Harvard Business School Publishing Corporation, 26-37.

López, J. P., \& Fierro, I. (2015). Determinantes de la felicidad en los ad- mistradores: una investiga- ción realizada en las farmacias del Grupo Difare en Ecuador. Universidad \& Empresa, 17(29), 181-211.

Lyubomirsky, S. (2005). La ciencia de la felicidad Un método probado para conseguir el bienestar. Buenos Aires: Ediciones Urano

Marks, N. (10 de 03 de 2015). La ciencia de la felicidad aplicada al trabajo. foro internacional. Quito, Ecuador.

Martínez, V., Moliner, C., Ramos, J., Luque, O., \& Gracia, E. (2014). Calidad y bienestar en organizaciones de servicios: el papel del clima de servicio y la justicia . Papeles del psicólogo, 35, 99-106.

Millán, A., \& Aubeterre, M. (2013).

Confirmación de la estructura factorial de la escala de disposición a fluir en el trabajo . Researchgate, 118-114.

Montoya Zuluaga, P. A., \& Moreno Moreno, S. (2012). Psicología desde el Caribe issn 0123-417X (impreso) ISSN 2011-7485 (on line) Vol. 29, n. ${ }^{\circ}$ 1, enero-abril 2012 relación entre síndrome de burnout, estrategias de afrontamiento y engagemen. Psicología desde el Caribe, 29, n. ${ }^{\circ} 1,206-227$.

Moreno Jiménez, B., Corso de Zúñiga, S., Sanz-Vergel, A. I., Rodriguez-Muñoz, A., \& Boada Pérez, M. (2010).

52

PODIUM No. 31, Guayaquil, Junio 2017, pp. 42-53

Universidad Espíritu Santo - UEES

ISSN: $1390-5473$ 
El "burnout" y el "engagement" en profesores de perú. Aplicación del modelo de demandas-recursos laborales. Copyright , 16(2-3), 293-307.

Ocampo Bustos, R. M., Juárez García, A., Arias Galicia, L. F., \& Hindrichs, I. (2015). Factores psicosociales asociados a engagement en empleados de un restaurante de morelos, México. Liberabit, 21(2), 207-219.

OMS. (2016). WWW.OMS.ORG. Obtenido de http://www.who.int/about/mission/es/: http://www.who.int/about/mission/es/

Orgambídez-Ramosa, A., Pérez-Morenoc, P. J., \& Borrego-Alés, Y. (2015). Estrés de rol y satisfacción laboral: examinando el papel mediador del engagement en el trabajo. Journal of Work and Organizational Psychology, 31, 69-77.

Prieto-Ursúa, M. (2006). Psicología Positiva: una moda polémica . Clínica y Salud, $17 n .^{\circ} 3,319-338$.

Salanova, M., Schaufeli, W., Llorens, S., Peiro, J., \& Grau, R. (2000). Desde el burnout al engagement: Una nueva perspectiva. Revista de psicología del trabajo y las organizaciones , 120-121.

Salanova, M., \& Schaufeli, W. B. (2009). El engagement en el trabajo. Madrid, España: Alianza editorial.
Salazar, J., \& Arenas, L. (2016). Un modelo económico de felicidad y su evidencia para México. Estudios Sociales 47, 24, 3-30.

Seligman, M. (2005). La auténtica felicidad. Buenos Aires, Argentina: Ediciones Byblos.

Sen, A. (1998). Capital humano y capacidad humana. Cuadernos de economía, 17, 67-72.

Valdés, M. (1991). Dos aspectos en el concepto de bienestar. Doxa: Cuadernos de filosofía del derecho., 9 , 69-89.

Vera, B. (2006). Psicología positiva: una nueva forma de entender la psicología . Papeles del Psicólogo Vol. 27(1), 3-8 .

Yakubova, D. (2016). Concepto de Bienestar en las lenguas Española y Rusa. Tonos digital, 1.

Zúñiga, C. (2008). La psicología positiva: un cambio en nuestro enfoque patológico clásico . Instituto de Investigación. Escuela Profesional de Psicología Universidad de San Martín de Porres , 82-88. 